\title{
Power-Aware Rendezvous with Shrinking Footprints
}

\author{
Hassan Jaleel and Magnus Egerstedt
}

\begin{abstract}
In this paper we investigate how power consumption affects mobility-based coordination algorithms for multirobot systems by explicitly coupling the control laws to the available power levels. In particular, we will consider a sensor network comprising of mobile sensors which use omni directional RF or radar based antennas for communication, with a disk-shaped communications footprint. As power decrease with time, the footprint shrinks as well, and in this paper we propose a controller that solves the rendezvous problem for such systems, thus providing a novel coupling between mobility algorithms and the available power levels.
\end{abstract}

\section{INTRODUCTION}

Distributed control and coordination of multiagent systems is a highly active area of research. Different control algorithms have been proposed that make the team of agents perform a wide variety of tasks, such as moving in formations (e.g., [2], [3], [6], [12]), establishing coverage and boundary protection (e.g., [1], [9]), and avoiding obstacles while maintaining group cohesion (e.g., [10], [17]). The basic concept behind these systems is that each agent has to make its own decisions, independent of the rest of the network. This decision is based solely on the local information the agent has access to about its surroundings and its neighboring agents, [9].

Despite the algorithmic advances made for coordinating mutliagent systems, there still remains a slight mismatch between the system models and the actual devices on which the algorithms will ultimately be deployed. In fact, mobile sensor networks typically consist of low cost and low power devices where each device is battery powered and batteries inevitably get depleted, i.e., their power levels decrease over time. Adding to this, different types of tasks consume different amount of power, with mobility typically being more costly than communications, e.g., [7].

In this paper, we take this decrease in power levels into account already at the controls design phase. For this to be meaningful, we first have to understand the effects the decreasing power levels have on the performance of the individual agents as well as on the entire system. Obviously, this depends on the type of devices being used. For example, if the system consists of vision based sensors, power levels may be related to the maximally available frame rate; for RF or radar based sensors, the footprint area may be reduced with the reduction in power; and in common communication networks, latency issues may arise across the network. To date, the coupling between power decay and mobility has

Authors are with the School of Electrical and Computer Engineering, Georgia Institute of Technology, Atlanta, GA 30332; Emails: \{hjaleel3, magnus\}@ece.gatech.edu. not been explicitly addressed in an integrated fashion, and in this paper we take a first stab at this issue by considering the so-called rendezvous problem (have all agents meet at the same location) based on RF or radar based sensors.

The rendezvous problem belongs to the class of consensus problems (see for example [4], [9], [10], [13], [14], [15], [16], [18]), where a collection of agents have to agree on some state value (position in this case). These problems are fundamental to the distributed control of multiagent systems, and they have been studied extensively for a large number of applications, including connectivity-based rendezvous [5], [19], formation control [2], [5], coverage control [1], just to name a few.

In this paper we add to this body of work by tying the control laws to the available power levels. As this constitutes a first step in this general research direction, we will restrict our analysis to sensors which use RF or radar based omni directional antennas for their communications. As a result, the network topology can be represented by a so-called timevarying $\Delta$-disk proximity graph, where $\Delta$ is the radius of the sensor footprint disk [1], [11]. Moreover we use a power model where power decay is independent of the mobility and is only a function of the current power level. The power decay will result in $\Delta$ being time-varying and the contribution in this paper is a controller that ensures that if we start with a connected network graph, the graph will remain connected and rendezvous will be achieved despite the reduction in sensor footprints. The actual control law is an extension to the control law proposed in [5], where edge weights are introduced for ensuring that more attention is given to edges (inter-agent interactions) that are close to becoming disconnected.

The outline of this paper is as follows. In Section II, we motivate the problem at hand with an example where the effect of shrinking footprints causes a "standard" coordination algorithm to fail. In Section III, we provide some basic definitions and introduce the tools and techniques from algebraic graph theory needed for the remainder of the paper. In Section IV, we introduce our general approach to addressing the rendezvous problem, which will be of a weighted consensus equation variety. This general approach will be specialized in Section $\mathrm{V}$ to the power aware rendezvous problem for the case of undirected graphs, which corresponds to the situation when all power levels in the network are the same. This (obviously somewhat simplistic) assumption is relaxed in Section VI, where we extend our results to directed graphs. 

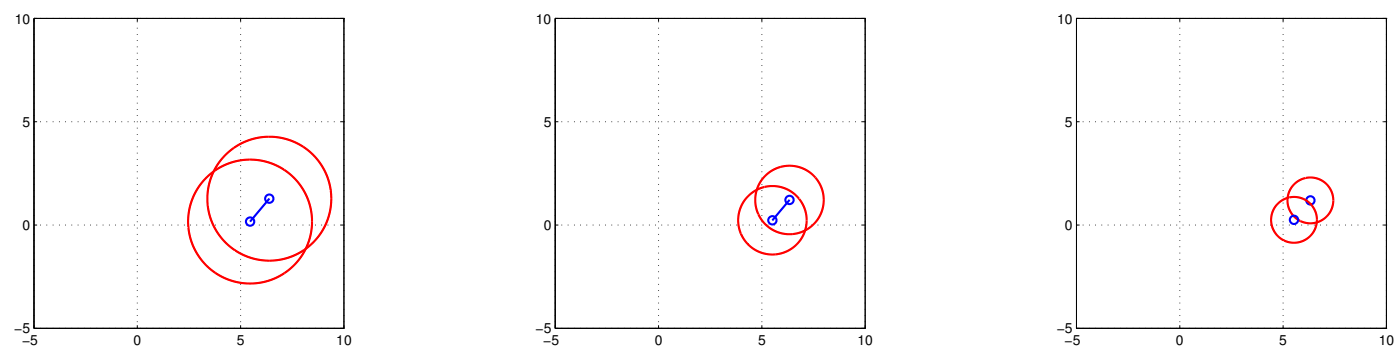

Fig. 1. Consensus algorithm on a two node network with shrinking disks. The parameters used in this simulation are, $\gamma=5$ and $\Delta(0)=3$. The straight line between the nodes in the first two figures indicate, the nodes are connected. However due to the shrinking footprints the network graph eventually gets disconnected in the last figure (no line between the two nodes).

\section{THE EFFECT OF SHRINKING FoOTPRINTS}

To establish some of the implications that shrinking sensor footprints have on the performance of the coordination algorithms, consider a system where each agent is a mobile sensing device that uses an omnidirectional antenna for communication. We define the footprint of a sensor as the region in which a sensor can detect any event and can communicate with other sensors. As such, we will use the terms sensor footprint and sensing region interchangeably. The footprint of these sensors is a disk of radius $\Delta$, and if we assume that $\Delta$ is fixed and same for all agents, we can represent the interaction topology with an undirected $\Delta$ disk graph (see [1], [9], [11]), where an edge exists between two nodes if the distance between them is less than or equal to $\Delta$. We will use the notation $N(i)$ to denote the index of all agents that are inside agent $i$ 's $\Delta$-disk. If we let the planar position of each agent be $x_{i} \in \mathbb{R}^{2}$, we can address the rendezvous problem using the consensus equation

$$
\dot{x}_{i}=-\sum_{j \in N(i)}\left(x_{i}-x_{j}\right) .
$$

From [13], [15], [17], [1] we know that the consensus equation asymptotically drives all the agents to the initial centroid $\bar{x}=\frac{1}{N} \sum_{i=1}^{N} x_{i}(0)$ (i.e., the rendezvous problem is solved) if the graph remains connected for all times.

Now, suppose that the agents are battery powered and, as a result, the power level decreases with time. Martin et al has shown in [7] that for the RF-based sensors under consideration in this paper, the area of the sensing region is directly related to the available power. Since the battery power is decreasing, the radius of the sensing disk also decreases resulting in a system with sensors having shrinking footprints. What if we want to achieve rendezvous in such a system? Is it possible? How is the shrinking of the footprints going to effect the system? Is the consensus equation still helpful in this scenario? All these questions, in spite of their importance in many real life systems, are still unanswered and in this paper we lay down a basic framework for the analysis of such systems.

For the sake of arguments, assume (this assumption will be relaxed in later sections) that the radius of the footprints decrease according to the following dynamics

$$
\dot{\Delta}(t)=-\gamma \Delta(t),
$$

with decay rate $\gamma>0$. What we get is an exponentially decaying footprint radius, and a question one can ask is whether the linear consensus equation in Equation (1) solves the rendezvous problem also in this case. Letting the footprints shrink according to Equation (2), Figure (1) shows a situation involving two mobile sensors where, although we started with a connected graph, the decaying power levels caused the footprint radius to shrink to a value where the distance between the two agents becomes greater than $\Delta(t)$. As a result, the edge between the agents is lost causing the graph to get disconnected, thus violating the fundamental condition for the convergence of the consensus algorithm. From this simple simulation it is thus concluded that the controller (1) does not work. The construction of a controller that does work is in fact the main contribution of this paper.

\section{Algebraic Graph Theory - Basics}

In this section we introduce some basic tools from algebraic graph theory taken mainly from ([8], [9]), which will be needed to formulate the problem and for the analysis in later sections. The basic object under consideration here is the graph $\mathcal{G}(V, E)$ with vertex set $V$ and edge set $E \subseteq V \times V$. If the edge set is unordered, i.e., $\left(v_{i}, v_{j}\right) \in E \Leftrightarrow\left(v_{j}, v_{i}\right) \in E$ we say that the graph is undirected, and an undirected graph is connected if there is a path between any two vertices. In the case of undirected graphs, connectivity is exactly the property we need to establish convergence in later sections, i.e., for solving the rendezvous problem.

If the edge set is not unordered, the graph is directed referred to as a digraph - and in this case, different types of connectivity related concepts will prove important: A digraph is

- weakly connected if its disoriented graph (the graph obtained by removing the direction from the edges) is connected;

- rooted out-branching if (1) it does not contain a directed cycle and (2) it has a vertex $v_{r}$ (root) such that for every other vertex $v_{i}$ there is directed path from $v_{r}$ to $v_{i}$; and

- balanced if $\operatorname{deg}^{i n}(v)=\operatorname{deg}^{\text {out }}(v), \forall v \in V$ (the number of edges going in to any node is the same as the number of edges going out).

Now, consider an undirected graph $\mathcal{G}(V, E)$. Associate an arbitrary orientation to its edges, $\sigma: E(\mathcal{G}) \rightarrow\{-1,1\}$, 
such that $\sigma(i, j)=-\sigma(j, i)$. This gives the new digraph $\mathcal{G}_{\sigma}(V, E, \sigma)$. Its incidence matrix, $\mathcal{I}=\left[e_{i j}\right]$ is defined as

$$
e_{i j}= \begin{cases}1 & \text { if } v_{i} \text { is the head of the edge } e_{j} ; \\ -1 & \text { if } v_{i} \text { is the tail of the edge } e_{j} \\ 0 & \text { otherwise. }\end{cases}
$$

Using the incidence matrix, the graph Laplacian can be defined as $\mathcal{L}=\mathcal{I I}^{T}$, which can be shown to be independent of the choice of orientation $\sigma$. The Laplacian, $\mathcal{L}$, is always symmetric and positive semi definite, and we let $\lambda_{1}, \lambda_{2}, \ldots, \lambda_{N}$ be the (non-negative and real) eigenvalues of $\mathcal{L}$, indexed such that $\lambda_{1} \leq \lambda_{2} \leq \cdots \leq \lambda_{N}$. The multiplicity of zero eigenvalues of $\mathcal{L}$ gives the number of connected components of the graph. As such, if we have a connected graph, the eigenvalues of $\mathcal{L}$ will be $0=\lambda_{1}<\lambda_{2} \leq \cdots \leq$ $\lambda_{N}$. The eigenvector corresponding to $\lambda_{1}$ is $\mathbf{1}$, where $\mathbf{1}$ denotes a vector with all the entries equal to 1 .

Now, suppose we have $N$ agents in $\mathbb{R}^{n}$ and let $x_{i}^{T}=$ $\left(x_{i, 1}, x_{i, 2}, \ldots, x_{i, n}\right)$ be the location of agent $i$. We can produce a new vector $c(x, j)$ as

$$
c(x, j)^{T}=\left(x_{1, j}, x_{2, j}, \ldots, x_{N, j}\right) .
$$

And, if we decompose the problem along each dimension, the consensus controller in Equation (1) becomes

$$
\dot{c}(x, j)=-\mathcal{L}(\mathcal{G}) c(x, j) \quad j=1,2, \ldots, n
$$

where $\mathcal{L}(\mathcal{G})$ is the laplacian matrix corresponding to graph $\mathcal{G}$.

In this paper, we will study graphs where the edge set changes (so-called dynamic graphs) as the agents move in and out of each others' sensory ranges. In particular, we will study $\Delta$-disk graphs where the vertex set $V=$ $\{1, \ldots, N\}$ corresponds to the indices of the different agents, and $\left(v_{i}, v_{j}\right) \in E \Leftrightarrow\left\|x_{i}-x_{j}\right\| \leq \Delta$, for some given $\Delta>0$. By definition, these graphs are undirected but will also need to introduce directed disk graphs where $\left(v_{i}, v_{j}\right) \in$ $E \Leftrightarrow\left\|x_{i}-x_{j}\right\| \leq \Delta_{j}$. The interpretation here is that information about agent $i$ will be available to agent $j$ if agent $i$ is no further than $\Delta_{j}$ away from agent $j$, where $\Delta_{j}>0$ is the sensor footprint radius associated with agent $j$.

\section{The Weighted Consensus Equation}

The controller in Equation (1) solves the rendezvous problem for undirected graphs as long as the graph remains connected for all times [4], [9]. But for $\Delta$-disk graphs (even without shrinking footprints), this condition on connectivity cannot be guaranteed, as shown in [5], [19]. The solution is to modify Equation (1) by introducing edge weights $w\left(x_{i}, x_{j}\right)$ that turn the linear consensus equation into a nonlinear equation

$$
\dot{x}_{i}=-\sum_{j \in N(i)} w\left(x_{i}, x_{j}\right)\left(x_{i}-x_{j}\right),
$$

where $w\left(x_{i}, x_{j}\right):\left(\mathbb{R}^{2}, \mathbb{R}^{2}\right) \rightarrow \mathbb{R}$ or, in matrix form

$$
\dot{c}(x, j)=-\mathcal{L}_{w} c(x, j) \quad \forall j=1,2, \ldots, n,
$$

where $\mathcal{L}_{w}$ is the weighted Laplacian matrix $\mathcal{L}_{w}=\mathcal{I W I}^{T}$, with $\mathcal{W}$ being a diagonal matrix of dimensions $(M \times M)$, where $M$ is the number of edges in the graph $\mathcal{G}$, and $w_{k k}=$ weight of the edge $\mathrm{k}$.

For the proper selection of these weights, define an edge tension energy along each edge $\left(v_{i}, v_{j}\right) \in E(\mathcal{G})$ as

$$
\begin{cases}\mathcal{E}_{i j}(x)>0 & \text { if }\left(v_{i}, v_{j}\right) \in E(G) \text { and } x_{i} \neq x_{j} ; \\ \mathcal{E}_{i j}(x)=0 & \left(v_{i}, v_{j}\right) \notin E(G)\end{cases}
$$

The total energy of the system becomes

$$
\mathcal{E}(x)=\sum_{i=1}^{N} \sum_{j=1}^{N} \frac{1}{2} \mathcal{E}_{i j}(x) .
$$

and in [5] it was shown that if the network topology is undirected, and

$$
\mathcal{E}_{i j}(x)=\frac{\left\|x_{i}-x_{j}\right\|^{2}}{\Delta-\left\|x_{i}-x_{j}\right\|} .
$$

then the controller

$$
\dot{x}_{i}=-{\frac{\partial \mathcal{E}}{\partial x_{i}}}^{T}=-\sum_{j \in N(i)} w_{i, j}\left(x_{i}, x_{j}\right)\left(x_{i}-x_{j}\right),
$$

ensures that graph remains connected $\forall t$ given that it was connected at $t=0$, while solving the rendezvous problem for $\Delta$-disk proximity graphs, with a fixed and time-invariant $\Delta$.

\section{RENDEZVOUS FOR UNDIRECTED GRAPH TOPOLOGIES WITH SHRINKING FOOTPRINTS}

The weighted consensus equation in Equation (8) works under the two crucial (and somewhat simplistic) assumptions that the radius of the sensor footprint disk, $\Delta$, is the same for all agents as well as constant in time. What we will do for the remainder of this paper is to relax these two assumptions, and we will start, in this section, by relaxing the assumption about a constant $\Delta$, while still insisting on the sensor disk radius being the same for all agents, which results in an undirected graph topology. This, second assumption will be relaxed in the next section, where the directed case will be treated.

Assuming that the agents use omni-directional RF based antennas for their communications, the size of the footprint $\Delta(t)$ decreases over time. We model this decrease as

$$
\dot{\Delta}=f(\Delta),
$$

with $f(\Delta)$ being a negative definite, Lipschitz continuous function, i.e., $f(\Delta)<0 \forall \Delta>0$, and $f(0)=0$. Since we assume that all agents have $\Delta$ as their sensory footprint disk radius, we have an undirected graph. The power decay model defined in Equation (9) depends only on the currently available power. This is obviously unrealistic because in any real life system, power model must also be a function of mobility. However, being a first attempt in this research direction, we have used a simplified model and plan to incorporate a more detailed and realistic model for future work. 
Starting with the same edge tension function as that in [5] (under the additional caveat that $\Delta$ is now a function of time), we get

$$
\mathcal{E}_{i j}(x)=\frac{\left\|d_{i j}\right\|^{2}}{\Delta(t)-\left\|d_{i j}\right\|},
$$

where $d_{i j}=x_{i}-x_{j}$.

Following the procedure in [5], we have

$$
\dot{\mathcal{E}}(t)=\frac{\partial \mathcal{E}}{\partial x} \dot{x}+N \frac{\partial \mathcal{E}}{\partial \Delta} \dot{\Delta}
$$

Letting $\dot{x}_{i}$ be given in Equation (8), we get

$$
\dot{\mathcal{E}}(t)=-\left\|\frac{\partial \mathcal{E}}{\partial x}\right\|^{2}-N\left(\frac{\left\|d_{i j}\right\|^{2}}{\left(\Delta-\left\|d_{i j}\right\|\right)^{2}}\right) f(\Delta) .
$$

We need to show that $\dot{\mathcal{E}}(t)<0$, for all t. Since $f(\Delta)$ is negative definite, this means the second term in the above expression has to be greater than $\left\|\frac{\partial \mathcal{E}}{\partial x}\right\|^{2}$ and it should be true for all possible $f(\Delta)$. For this to be true the following condition should always hold

$$
|f(\Delta)| \leq \frac{2 \Delta-\left\|d_{i j}\right\|}{\left(\Delta-\left\|d_{i j}\right\|\right)^{2}}
$$

The right side of the expression is a function of distance between the agents and $\Delta$ while the left side is function of $\Delta$ only. Hence it is clear we can not guarantee this energy is always decreasing (which is the key to the convergence argument) for all negative definite $f(\Delta)$. As such we need to modify our controller. .

Lemma 5.1: Given an undirected $\Delta$-disk graph $\mathcal{G}(V, E)$ which is connected at $t=0$ in such a way that $\| x_{i}(0)-$ $x_{j}(0) \|<(\Delta(0)-\epsilon)$, for some $\epsilon>0$ for all $\left(v_{i}, v_{j}\right) \epsilon$ $E(0)$. Then, under the control law

$$
\dot{x}_{i}=-\left({\frac{\partial \mathcal{E}^{T}}{\partial x_{i}}}^{T}+{\frac{\partial \mathcal{E}}{\partial x_{i}}}^{T} \frac{\partial \mathcal{E}}{\partial \Delta} \frac{\dot{\Delta}}{\left\|\frac{\partial \mathcal{E}}{\partial x_{i}}\right\|^{2}}\right),
$$

the graph $\mathcal{G}(V, E, \Delta)$ remains connected $\forall t>0$.

Proof: Consider the energy function in Equation (7). Then

$$
\dot{\mathcal{E}}=\frac{\partial \mathcal{E}}{\partial x} \dot{x}+N \frac{\partial \mathcal{E}}{\partial \Delta} \dot{\Delta} .
$$

Using $\dot{x}_{i}$ as defined in Equation (11), we obtain

$$
\frac{\partial \mathcal{E}}{\partial x} \dot{x}=-\left\|\frac{\partial \mathcal{E}}{\partial x}\right\|^{2}-N\left(\frac{\partial \mathcal{E}}{\partial \Delta} \dot{\Delta}\right),
$$

and as such

$$
\dot{\mathcal{E}}=-\left\|\frac{\partial \mathcal{E}}{\partial x}\right\|^{2} \leq 0 \quad \forall t>0 .
$$

What this implies is that the energy in the system is never increasing. But, the energy approaches infinity as edge lengths approach $\Delta$, this can never happen. In particular, suppose on the contrary that there exists an edge $\left(v_{i}, v_{j}\right)$ such that at some time $t$ the corresponding length is $\Delta(t)$. We know that at $t=0$ the edge length is less than $(\Delta(0)-\epsilon)$ and the total energy $\mathcal{E}(0)$ of the system defined in Equation (6) is finite. But, if at time $\hat{t}$, the edge length is equal to $\Delta(\hat{t})$ then the energy is $\mathcal{E}(\hat{t})=\infty$, meaning that $\mathcal{E}(\hat{t})>\mathcal{E}(0)$, which is a contradiction, and the lemma follows.

Theorem 5.2: Given an undirected $\Delta$-disk graph which is connected at $t=0$ with edge lengths less than $(\Delta(0)-$ $\epsilon$ ) for some $\epsilon>0$. Under the controller in Equation (11), the system converges asymptotically to the initial centroid of the network.

Proof: Rearranging the terms in Equation (11) gives

$$
\dot{x}_{i}=-\sum_{j \in N(i, t)}\left[1+\frac{\partial \mathcal{E}}{\partial \Delta} \frac{\dot{\Delta}}{\left\|\frac{\partial \mathcal{E}}{\partial x_{i}}\right\|^{2}}\right] \frac{\partial \mathcal{E}_{i j}{ }^{T}}{\partial x_{i}},
$$

where $N(i, t)$ is the set of neighbors to agent $i$ at time $t$. This expression can be written on the standard, weighted consensus form

$$
\dot{x}_{i}=-\sum_{j \in N(i, t)} w_{i j}\left(x_{i}, x_{j}\right)\left(x_{i}-x_{j}\right),
$$

with

$$
w_{i j}(t)=\left[1+\frac{\partial \mathcal{E}}{\partial \Delta} \frac{\dot{\Delta}}{\left\|\frac{\partial \mathcal{E}}{\partial x_{i}}\right\|^{2}}\right] \frac{2 \Delta-d_{i j}}{\left(\Delta-d_{i j}\right)^{2}} .
$$

We can also write this controller in terms of a weighted Laplacian.

$$
c(x, j)=-\mathcal{L}_{w} c(x, j) \forall j=1,2, \ldots, n
$$

Now, it is known [5], that this controller (15) drives all the agents asymptotically to initial centroid as long as the network stays connected. But connectivity follows from Lemma 5.1, and the proof follows.

What this new controller allows us to do is to compensate for the shrinking footprints by explicitly taking this into account already at the control design phase. Figure (2) shows a Matlab simulation of controller (13) with weight function (14) for an undirected graph. However, we still assume that all the footprints are the same. What remains to do is to investigate what happens if they are no longer the same, which is the topic in the next section.

\section{RENDEZVOUS FOR DiRECTED GRAPH TOPOLOGIES WITH SHRINKING FOOTPRINTS}

In this section, we consider the case when the footprints are still decaying according to

$$
\dot{\Delta}_{i}=f\left(\Delta_{i}\right)
$$

but where we no longer assume that $\Delta_{i}=\Delta_{j}$, i.e., when the footprints are no longer the same. As a result, we have a directed graph since if $\left\|d_{i j}\right\| \leq \Delta_{j}$, this certainly does not imply that $\left\|d_{i j}\right\| \leq \Delta_{i}$. As such, we need to show that our previously defined controller works for directed graph topologies as well. 

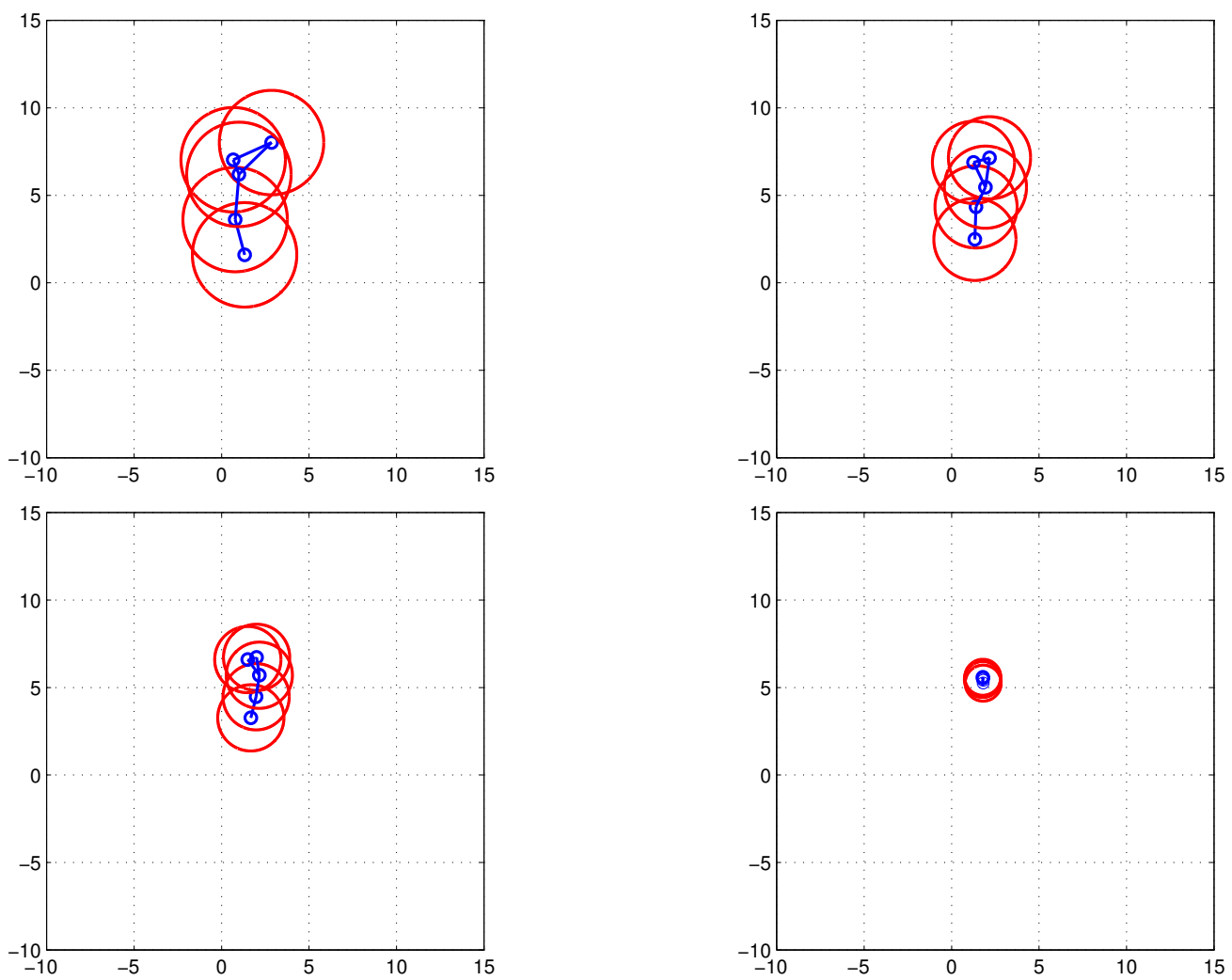

Fig. 2. Consensus algorithm (11) on a 5 node undirected network with shrinking disks according to (2). $\gamma=5, \Delta(0)=3, t_{1}=0.0010$ sec, $t_{2}=0.0490$ sec, $t_{3}=0.0920 \mathrm{sec}, t_{4}=0.2140 \mathrm{sec}$.

Theorem 6.1: Given a directed disk graph $\mathcal{G}(V, E)$ with $\bar{\Delta}(t)^{T}=\left[\Delta_{1}(t), \ldots, \Delta_{N}(t)\right]$. If the initial graph is balanced and weakly connected and all initial edges $\left(v_{i}, v_{j}\right)$ have edge lengths less that $\min _{i} \Delta_{i}(0)-\epsilon$ for some $\epsilon>0$, then the control law

$$
\dot{x}_{i}=-\sum_{j \in N(i, 0)} w_{i j}\left(x_{i}, x_{j}\right)\left(x_{i}-x_{j}\right)
$$

with weight function $w_{i j}$ given as

$$
w_{i j}(t)=\left[1+\frac{\partial \mathcal{E}}{\partial \Delta_{i}} \frac{\dot{\Delta}_{i}}{\left\|\frac{\partial \mathcal{E}}{\partial x_{i}}\right\|^{2}}\right] \frac{2 \Delta_{i}-d_{i j}}{\left(\Delta_{i}-d_{i j}\right)^{2}} .
$$

makes the multiagent system converge asymptotically to initial centroid, i.e., the rendezvous problem is solved

Proof: From the results proved in [9] we know that the agreement protocol $\dot{x}=-\mathcal{L}_{w} x$ over directed graph reaches average consensus if and only if the directed graph is weakly connected and balanced. We have already shown in the previous section that we can write our controller in terms of a weighted Laplacian (15), and also that this controller does not loose any edges. And, by making the neighborhood set be equal to the initial neighborhood set $(N(i, 0))$, as long as we start with a balanced and weakly connected graph then these characteristic will be maintained and thus all the agents will drive asymptotically to initial centroid. This concludes the proof.

What this implies is that we indeed have solved the rendezvous problem also for shrinking footprints, which is what one must do when taking decaying power levels into account. An example of this is shown in Figure 3.

If we analyse the weight function (14) more closely there is a potential problem. Consider two agents, $i$ and $j$, which are not neighbours initially. Suppose at some time $t$ they come at a distance exactly equal to $\Delta(t)$ from each other and due to $\Delta$-disk graph topology, immediately become neighbours with the edge tension energy as is given in Equation (7). However at this moment $\left\|d_{i j}\right\|=\Delta(t)$, which makes the energy $\infty$. To avoid this situation one simple solution is to introduce hysteresis. We define a function $h_{i j}$ such that

$$
h_{i j}= \begin{cases}1, & \left\|d_{i j}\right\| \leq(\Delta-\epsilon) \\ 0 & \text { otherwise. }\end{cases}
$$

So adding this aspect to (8), we get the following controller

$$
\dot{x}_{i}=-\sum_{j=1}^{N} h_{i j} w_{i j}\left(x_{i}-x_{j}\right) \text {. }
$$

\section{CONCLUSION}

In this paper we considered the scenario in which the sensor footprints associated with a mobile sensor networks 

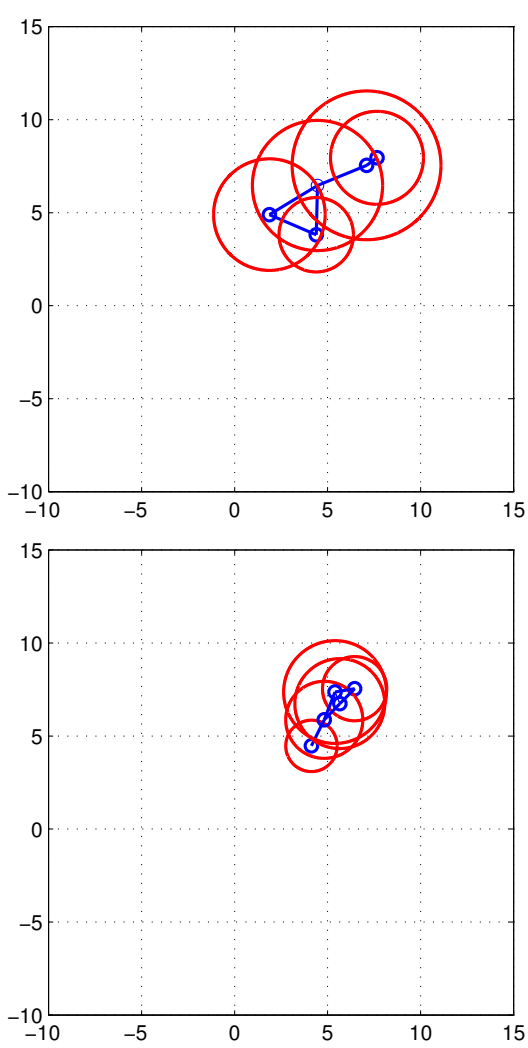
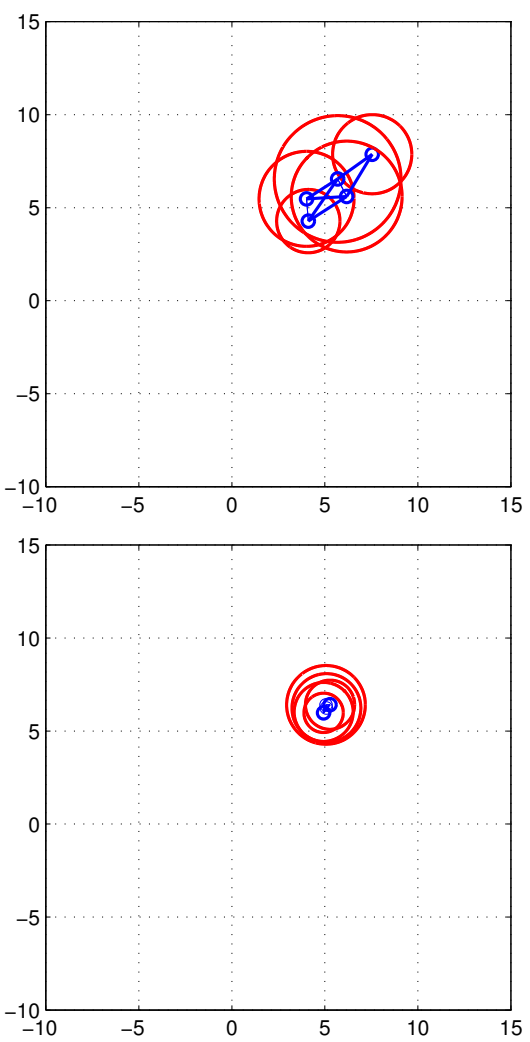

Fig. 3. Consensus algorithm (11) on a 5 node directed network with shrinking disks according to (2). $\gamma=5, \bar{\Delta}(0)=[2,2.5,3,3.5,4], t_{1}=0.0010$ sec, $t_{2}=0.0323 \mathrm{sec}, t_{3}=0.0741 \mathrm{sec}, t_{4}=0.1275 \mathrm{sec}$.

are shrinking over time. We proposed a controller that solve rendezvous problem, which constitutes the first attempt to explicitly couple mobility algorithms with power decay models already at the design stage.

\section{REFERENCES}

[1] J. Cortés, S. Martinez, and F. Bullo, ”Robust rendezvous for mobile autonomous agents via proximity graphs in d dimension," in IEEE Trans. Robot. Autom., vol. 51, no. 8, Aug. 2006, pp. 1289 -1298.

[2] J. Desai, J. Ostrowski, and V. Kumar, "Controlling formations of multiple mobile robots," in Proc. IEEE Int. Conf. Robot. Autom., Leuven, Belgium, 1998, pp. 2864 - 2869.

[3] M. Egerstedt and X. Hu, "Formation constrained multi-agent control," in IEEE Trans. Robot. Autom., vol. 17, no. 6, Dec. 2001, pp. 947 951.

[4] A. Jadbabaie, J. Lin, and A. S. Morse, "Coordination of groups of mobile autonomous agents using nearest neighbor rules," in IEEE Trans. Autom. Control, vol. 48, no. 6, Jun. 2003, pp. 988 - 1001.

[5] M. Ji and M. Egerstedt, "Distributed Coordination Control of Multiagent Systems While Preserving Connectedness", in IEEE Trans. on Robotics, vol. 23, issue 4, Aug. 2007, pp. 693 - 703.

[6] N. E. Leonard and E. Fiorelli, "Virtual leaders, artificial potentials and coordinated control of groups," in Proc. IEEE Conf. Decision Control, Orlando, FL, Dec. 2001, pp. 2968 - 2973.

[7] P. Martin, R. Galvan-Guerra, and M. Egerstedt. "Power-Aware Sensor Coverage: An Optimal Control Approach," in $19^{\text {th }}$ International Symposium on Mathematical Theory of Networks and Systems MTNS, Budapest, Hungary, July 2010.

[8] C. Godsil and G. Royle, Algebraic Graph Theory, Berlin, Germany, Springer, 2001.

[9] M. Mesbahi and M. Egerstedt, Graph Theoretic Methods for Multiagent Networks, Princeton University Press, Princeton, NJ, Sept. 2010.
[10] L. Moreau, "Stability of multiagent systems with time-dependent communication links," in IEEE Trans. Autom. Control, vol. 50, no. 2, Feb. 2005, pp. $169-182$.

[11] A. Muhammad and M. Egerstedt, "On the structural complexity of multiagent robot formations," in Proc. Am. Control Conf. 2004, Boston, MA, 2004, pp. 4957 - 4962.

[12] P. Ögren, M. Egerstedt, andX.Hu, "A control Lyapunov function approach to multi-agent coordination," in IEEE Trans. Robot. Autom., vol. 18 , no. 5, Oct. 2002, pp. 847 - 851

[13] W. Ren and R. Beard, "Consensus of information under dynamically changing interaction topologies", in Proc. Am. Control Conf., vol. 6 , Jun. 30Jul. 2, 2004, pp. 4939 - 4944.

[14] C. Reynolds, "Flocks, herds and schools: A distributed behavioral model," in Proc. ACM SIGGRAPH 1987 Conf., 1987, vol. 21, no. 4, pp. $25-34$.

[15] R. O. Saber and R. M. Murray, "Agreement problems in networks with directed graphs and switching toplogy," in Proc. 42nd IEEE Conf. Decision Control, vol. 4, Maui, HI, Dec. 2003, pp. 4126 - 4132.

[16] R. O. Saber and R. M. Murray, "Flocking with Obstacle Avoidance: Cooperation with Limited Communication in Mobile Networks", in Proc. 42nd IEEE Conf. Decision Control, vol. 2, Maui, HI, Dec. 2003, pp. 2022 - 2028.

[17] R. O. Saber and R. M. Murray, "Consensus problems in networks of agents with switching topology and time-delays", in IEEE Trans. Autom. Control, vol. 49, no. 9, Sep. 2004, pp. 1520 - 1533.

[18] T. Vicsek, A. Czirok, E. B. Jacob, I. Cohen, and O. Schochet, "Novel type of phase transitions in a system of self-driven particles," in Phys. Rev. Lett., vol. 75, 1995, pp. 1226 - 1229.

[19] M. M. Zavlanos, H. G. Tanner, A. Jadbabaie and G. J. Pappas. Hybrid Control for Connectivity Preserving Flocking. IEEE Transactions on Automatic Control, Vol. 54, No. 12, pp. 2869-2875, December 2009. 